\title{
Translation Invariance and Instability of Phase Coexistence In the Two Dimensional Ising System
}

\author{
Michael Aizenman` \\ Department of Physics, Princeton University, Princeton, NJ 08544, USA
}

\begin{abstract}
It is shown that any Gibbs state of the two dimensional ferromagnetic Ising system is of the form $\lambda \mu_{+}+(1-\lambda) \mu_{-}$, with some $\lambda \in[0,1]$. This excludes the possibility of a locally stable phase coexistence and of translation symmetry breaking, which are known to occur in higher dimensions. Use is made in the proof of the stochastic aspects of the geometry of the interface lines.
\end{abstract}

\section{Introduction}

The collective phenomena exhibited by systems of locally interacting spins on infinite lattices are of considerable interest for diverse subjects, including statistical mechanics, field theory and solid state physics. Of special interest is the behavior at phase transitions, the simplest of which correspond to situations in which the system may be at a thermodynamic equilibrium in more than one state.

When there is no unique equilibrium state one may frequently identify a number of pure phases, which are states with certain homogeneity. The following discussion bears on the possibility of a locally-stable coexistence of distinct phases. This would be described by a state in which, while thermodynamic equilibrium is maintained, in different regions the typical configurations show behavior characteristic of different pure phases. In some cases, as the one discussed below, the coexistence may be described by the presence of a sharply defined interface. If both the interactions and the pure phases are translation invariant, the phase coexistence relates to the possibility of the breaking of translation symmetry.

It has been generally expected that for systems with short range interactions the possibility of such a phase coexistence depends on the dimension of the lattice. For the dimension three and more Dobrushin proved in 1972 [1] that coexistence is possible in the Ising system at low temperatures. The coexistence was induced there by mixed boundary conditions on a sequence of increasing cubes, - on the

* $\quad$ Supported in part by the U.S. National Science Foundation under the grant PHY - 7825390 
upper halves and + on the lower halves of the surfaces. The proof that for these states translation symmetry in the vertical direction is broken was much simplified by van Beijern [5]. The construction was recently extended to the WidomRowlinson lattice model [6].

For the analogous boundary conditions in two dimensions, Gallavotti proved that at low temperatures the limiting state is a convex combination (ensemble average) of the pure phases [8]. Subsequently, this result was extended to all the temperatures and a collection of other boundary conditions $[9,10,11]$, indicating that these may be the only Gibbs states of the two dimensional system. The best evidence in this direction has been a recent proof, by Russo [13], of such an assertion for all the Gibbs states which have one of the main symmetries of the lattice.

The main result described here finally settles the question for the two dimensional Ising system. In this note we provide details of the proof, which was announced in [14], that at any temperature below $T_{c}$ the only Gibbs states of the system are convex combinations of the pure phases $\mu_{+}$and $\mu_{-}$. In particular, this excludes the possibility of the translation-symmetry breaking.

In the analysis, which can generally be described as stochastic-geometrical, we first identify an interface which is associated with phase coexistence. This provides a characterization of any given spin configuration as a patchwork of several purephase components, enabling us to reduce the study of equilibrium states of the system of spins to that of a system of lines. We then prove that there is no Gibbs state which corresponds to a stable coexistence of two phases with a single interface which is in some sense "flat". This part of the proof depends essentially on the availability of contour arguments and the FKG inequality. The same method may be used to show that there is no Gibbs state with any finite number of interfaces, winding finitely around the origin. Finally, it is shown that in any Gibbs state there can be no more than one interface and that it has the properties which were assumed in the previous step.

Since the submittal and circulation of the announcement [14] partial results in this direction were also given in [15] and the main result was given another proof by Higuchi [16]. In our method, however, the reference to inequalities other than FKG occurs only in the last step, which for low temperatures may be proven by a Peierls argument combined with the stronger version of the second step described above. Thus the following proof may be extended to show the instability of phase coexistence in some other two-phase systems, like the Widom-Rowlinson lattice model, at low temperatures.

\section{Preliminaries}

The two dimensional ferromagnetic Ising system (f.I.s) is described by the "spin" variables $\left\{\sigma_{i}\right\}_{i \in \mathbb{Z}^{2}}$ which take the values \pm 1 with equal, and independent, a-priori probabilities. Spin configurations are identified with points in the set $\Omega=\{-1,+1\}^{\mathbb{Z}^{2}}$.

For any $\Lambda \subset \mathbb{Z}^{2}$, we denote by $\sigma_{\Lambda}$ the restriction of a configuration $\sigma \in \Omega$ to $\Lambda$. $\mathfrak{B}_{\Lambda}$ is the $\sigma$-algebra, of subsets of $\Omega$, which is generated by such a restriction. 
$\mathfrak{B}_{\infty} \equiv \bigcap_{\Lambda \in P\left(\mathbb{Z}^{2}\right)} \mathfrak{B}_{\Lambda^{c}}$, is the $\sigma$-algebra of events "measurable at infinity", with $P\left(\mathbb{Z}^{2}\right)$ being the collection of finite subsets of $\mathbb{Z}^{2}$ and $\Lambda^{c} \equiv \mathbb{Z}^{2} \backslash \Lambda$.

States of the system are probability measures, $\mu$, on $\Omega$ (or rather on $\mathfrak{B}$ $\left.=\overline{\bigcup_{\Lambda \in P\left(\mathbb{Z}^{2}\right)} \mathfrak{B}_{\Lambda}}\right)$. We denote their restrictions to $\Lambda$ by $\mu_{\Lambda}(\cdot)$, the conditional expectations by $\mu\left(\cdot \mid \sigma_{A}\right) \equiv \mu\left(\cdot \mid \mathfrak{B}_{A}\right)(\sigma)$, or $\mu(\cdot \mid A)$ for $A \in \mathfrak{B}$, and integrals by $\mu(f)$.

The spins interact ferromagnetically, with the Hamiltonian

$$
H_{\Lambda}(\sigma) \equiv H\left(\sigma_{\Lambda} \mid \sigma_{\Lambda^{c}}\right)=-\frac{1}{2} \sum_{\substack{i, j \in A \\|i-j|=1}} \sigma_{i} \sigma_{j}-\sum_{\substack{i \in \Lambda, j \in \Lambda^{c} \\|i-j|=1}} \sigma_{i} \sigma_{j} .
$$

For an inverse-temperature $\beta$ the thermodynamic equilibrium of a state $\mu$ is characterized by the Dobrushin-Lanford-Ruelle (DLR) condition [2]:

$$
\mu_{\Lambda}\left(\left\{\sigma_{A}\right\} \mid \eta_{\Lambda^{c}}\right)=\exp \left[-\beta H\left(\sigma_{\Lambda} \mid \eta_{\Lambda^{c}}\right)\right] / \operatorname{Norm} .\left(\eta_{\Lambda^{c}}\right)
$$

which is the defining property of Gibbs states.

The study of the thermodynamic equilibrium is thus reduced to that of the collection of the system's Gibbs states, which we denote by $\Delta(\beta)$, with $\Delta=\bigcup_{\beta \geqq 0} \Delta(\beta)$. It is well known that for $\beta>\beta_{c}$ the (translation invariant) limits, $\mu_{+, \beta}, \mu_{-, \beta} \in \Delta(\beta)$, of finite volume Gibbs ensembles with +1 , correspondingly -1 , boundary conditions are distinct.

While on general grounds $\Delta(\beta)$ is closed under convex combination, we shall prove as our main result:

Theorem 1. For the two dimensional ferromagnetic Ising system at any $\beta \in[0, \infty)$ :

$$
\begin{aligned}
\Delta(\beta) & =\left\{\lambda \mu_{+, \beta}(\cdot)+(1-\lambda) \mu_{-, \beta}(\cdot)\right\}_{\lambda \in[0,1]} \\
& \equiv \operatorname{span}\left\{\mu_{+, \beta}, \mu_{-, \beta}\right\} .
\end{aligned}
$$

A very useful tool for our analysis is provided by the following general property of Gibbs states [2]:

Proposition 1. Let $\operatorname{Ext} \Delta(\beta)$ be the set of extremal elements of $\Delta$ (with respect to the convex combinations). Then $\forall \mu \in \Delta(\beta)$ :

(1) (Decomposition to extremal elements)

$$
\mu(\cdot)=\int_{\alpha \in I} \tilde{\mu}(d \alpha) v_{\alpha}(\cdot)
$$

for some measure $\tilde{\mu}$ on an index space I and some $v: I \rightarrow \operatorname{Ext} \Delta(\beta)$. (In fact, $\mu$-almost surely $\mu\left(\cdot \mid \mathfrak{B}_{\infty}\right)(\sigma) \in \operatorname{Ext} \Delta(\beta)$ and one may choose $I=\Omega, \tilde{\mu}=\mu$.)

(2) $\mu \in \operatorname{Ext} \Delta(\beta)$ if and only if $\forall B \in \mathfrak{B}_{\infty}: \mu(B)$ is either 0 or 1 .

(3) If $B \in \mathfrak{B}_{\infty}$ and $\mu(B) \neq 0$ then $\mu(\cdot \mid B) \in \Delta(\beta)$.

For two measures on $\Omega$ (or $\Omega_{A}$ ), $\mu_{\mathrm{FKG}} \geqq \mu$ means that $\mu(f) \geqq v(f)$ for any real function which is monotone with respect to the following partial order of $\Omega$ :

$$
\sigma \geqq \sigma^{\prime} \Leftrightarrow \sigma_{i} \geqq \sigma_{i}^{\prime} \forall i \in \mathbb{Z}^{2} .
$$


A more specific property of the Ising ferromagnetic system is that it satisfies the conditions required for the FKG inequality $[3,4]$. In particular, we shall use its following consequence.

Proposition 2[3]. Let $\eta, \xi \in \Omega_{\Lambda^{c}}$. If $\eta \geqq \xi$, then the Gibbs states of the f.I.s which are induced in $\Omega_{\Lambda}$ with $\eta$ and $\xi$ as the boundary conditions (for some fixed $\beta$ ) satisfy:

$$
\mu_{\Lambda, \eta} \underset{\mathrm{FKG}}{\geqq} \mu_{\Lambda, \xi} .
$$

We use $\left(i_{x}, i_{y}\right),\left(j_{x}, j_{y}\right)$ to denote the Cartesian coordinates of general $i, j \in \mathbb{Z}^{2}$. We shall also employ the following geometrical terminology and notation:

$$
\pi_{( \pm)}=\left\{i \in \mathbb{Z}^{2} \mid i_{x} \gtreqless 0\right\} \text {. }
$$

$i, j \in \mathbb{Z}^{2}$ are connected if $\left|i_{x}-j_{x}\right|+\left|i_{y}-j_{y}\right| \leqq 1$. The same points are $*$ connected if $\max \left\{\left|i_{x}-j_{x}\right|,\left|i_{y}-j_{y}\right|\right\} \leqq 1$, and / connected if in addition $\left(i_{x}-j_{x}\right)\left(i_{y}-j_{y}\right) \geqq 0$. of $\mathbb{Z}^{2}$.

A cluster $(*$ cluster or $/$ cluster $)$ : a connected $(*$ connected or $/$ connected) subset

An $\infty+$ cluster $(\infty$-cluster, etc): a cluster of infinite size on which $\sigma=+1(-1$, etc. $)$.

A contour of $\sigma$ : a polygon which connects nearest neighboring points of $\left(\mathbb{Z}+\frac{1}{2}\right) \times\left(\mathbb{Z}+\frac{1}{2}\right)$, and which separates two adjacent /clusters of opposite signs. It is an $\infty$ contour if its size is infinite. When referring to the number of contours in a region we shall, of course, count only the complete contours, which have no ends in the region.

The reason for our interest in the diverse notions of connectedness is that while a + cluster is not necessarily surrounded by a - cluster its exterior boundary is always a - *cluster. Further, the above definition of contours leads to non crossing lines. The use of /clusters amounts to a procedure of "rounding the corners" used to separate contours whenever four segments meet at a point.

Finally, a statement is true $\mu$-almost surely, or for $\mu$-a.e. $\sigma$. (" $\mu$ almost every $\sigma$ "), if the subset of $\Omega$ on which it is false has zero $\mu$-measure.

\section{Identification of the Interface}

Our first step in the analysis of phase coexistence is to identify a simple feature, with the aid of which we shall separate in general configurations regions of distinct phases. While its choice is somewhat arbitrary (since even in the pure phases locally no spin configuration is excluded) such an interface should satisfy the following three conditions.

(i) For a given configuration of the interface lines, the state in each of the regions which they separate should resemble a pure phase.

(ii) No interface line should be found in configurations which are typical for pure phases.

(iii) The absence of interface lines in configurations which are typical for some Gibbs state should imply that the state is a convex combination of the pure phases.

Of main interest for us is, of course, property (iii), which will provide a sufficient condition. However this condition would have been useless had (ii) been invalid. 
Natural candidates for interface lines are the infinite contours. Since they partition the space to regions with boundary spins of definite signs, (i) is clearly satisfied. For temperatures in the two phase region, the second condition is also met, as a consequence of the following result of L. Russo:

Proposition 3 (Proposition 1 of [13]). For the two dimensional f.I.s and $\beta>\beta_{c}$, in $\mu_{+, \beta}$-a.e. $\sigma$ and $\mu_{-, \beta}$-a.e. $\sigma$ there are no $\infty^{*}$ clusters of the opposite signs.

We shall adopt the above choice since it satisfies also condition (iii), as stated in the following proposition.

Proposition 4. Let $\mu \in \Delta(\beta)$. If $\mu$-a.e. $\sigma$ has no $\infty$ contour then $\mu \in \operatorname{span}\left\{\mu_{+, \beta}, \mu_{-, \beta}\right\}$.

Before proving Proposition 4 let us remark that Proposition 3 was proven by showing that, in $d=2$, the infinite + cluster of $\mu_{+}$forms a mesh which completely surrounds any region, preventing the existence of infinite $-{ }^{*}$ clusters (and thus of infinite contours).

To prove Proposition 4 we recall from [13]:

Lemma 1. Let $\mu \in \Delta(\beta)$. If $\mu$-almost surely there is no $\infty+$ cluster then $\mu=\mu_{-}$.

For the sake of completeness let us remark that Lemma 1 is a quick consequence, due to the Markov property and the FKG inequality, of the fact that the assumption implies that any finite volume is $\mu$-a.s. surrounded by a -cluster.

Proof of Proposition 4. Let $I_{i}$ be the characteristic functions, with values 0 or 1 , defined by the following mutually exclusive and exhausting events:

$I_{1}(\sigma)=1 \Leftrightarrow \sigma$ has no $\infty$ - clusters

$I_{2}(\sigma)=1 \Leftrightarrow \sigma$ has an $\infty$-clusters but no $\infty+$ clusters

$I_{3}(\sigma)=1 \Leftrightarrow \sigma$ has both an $\infty$-cluster and an $\infty+$ cluster.

If $\mu$ satisfies the assumptions in Proposition 1 , then $I_{3}(\sigma)=0$, i.e. $I_{1}(\sigma)$ $+I_{2}(\sigma)=1$, for $\mu$-a.e. $\sigma$, since the presence of two $\infty$ clusters of opposite signs implies the existence of an infinite contour. The function $I_{k}$ are measurable at infinity. Therefore, by Proposition 1 , whenever $\mu\left(I_{i}\right) \neq 0$ then $\frac{\mu\left(I_{i} \cdot\right)}{\mu\left(I_{i}\right)}$ is a Gibbs state. Applying Lemma 1 to these states, we get

$$
\begin{aligned}
\mu(\cdot) & =\mu\left(I_{1} \cdot\right)+\mu\left(I_{2} \cdot\right) \\
& =\mu\left(I_{1}\right) \mu_{+}(\cdot)+\mu\left(I_{2}\right) \mu_{-}(\cdot),
\end{aligned}
$$

which proves the claim.

\section{Instability of a Single Interface}

Let

$\Omega_{1}=\{\sigma \in \Omega \mid$ in $\sigma$ there is exactly one $\infty$ contour, $\gamma(\sigma)$, and $\gamma(\sigma)$ has a finite but non-empty intersection with each of the lines $\left\{i_{x}=\right.$ const $\}$. $\}$.

According to our interpretation, $\Omega_{1}$ includes the set of configurations with a single interface which is basically horizontal. Intuitively, that seems to be the simplest mode of phase coexistence. In this section we shall prove that for $d=2$, it 
is unstable at any non-zero temperature, in the sense given by the following proposition.

\section{Proposition 5. Let $\mu \in \Delta$. Then}

$$
\mu\left(\Omega_{1}\right)=0 .
$$

In the proof we shall use the "fluctuations at infinity" of the interface line. These are reflected in the following two lemmas which refer to "duplicate systems".

Lemma 2. Let $\mu \in \operatorname{Ext} \Delta$. If $\mu\left(\Omega_{1}\right)=1$ then for $\mu \times \mu$-a.e. $\left(\sigma, \sigma^{\prime}\right) \in \Omega \times \Omega$ the two contours $\gamma=\gamma(\sigma)$ and $\gamma^{\prime}=\gamma\left(\sigma^{\prime}\right)$ intersect infinitely often in both $\pi_{+}$and $\pi_{-}$.

Proof. Both $\gamma$ and $\hat{\gamma}$ have a finite intersection with the " $y$-axis", $\left\{i_{x}=0\right\}$, and have unique infinite connected components in $\pi_{+}$. Thus $\gamma$ defines a partition of $\pi_{+}$to two sets whose points may naturally be refered to as lying "above" or "below" $\gamma$.

The claim follows from the fact that the subset of $\Omega_{1} \times \Omega_{1}$ on which it is violated is a disjoint union of two sets of equal $\mu \times \mu$ probabilities, which are measurable at infinity. The sets are:

$$
\begin{aligned}
& A=\left\{\left(\sigma, \sigma^{\prime}\right) \in \Omega_{1} \times \Omega_{1} \mid \gamma^{\prime} \text { eventually lies "above" } \gamma \text { in } \pi_{+}\right\} \\
& B=\left\{\left(\sigma, \sigma^{\prime}\right) \in \Omega_{1} \times \Omega_{1} \mid \gamma^{\prime} \text { eventually lies "below" } \gamma \text { in } \pi_{+}\right\} .
\end{aligned}
$$

The equality of their probabilities is implied by the symmetry $\left(\sigma, \sigma^{\prime}\right) \rightarrow\left(\sigma^{\prime}, \sigma\right)$ of $\mu \times \mu$. Hence $\mu(A)=\mu(B) \leqq 1 / 2$. However the extremality of $\mu$ implies the triviality for $\mu$, and thus for $\mu \times \mu$, of the corresponding $\sigma$-algebras of "events measurable at infinity", by which $\mu(A)=\mu(B)$ is either 0 or 1 . Therefore

$$
\mu(A)=\mu(B)=0 \text {. }
$$

Let $T: \Omega \rightarrow \Omega$ be the shift defined by $(T \sigma)_{i}=\sigma_{i-(0,1)}$. For any measure $\mu$ we denote $\hat{\mu}(\cdot)=T \mu(\cdot)$ [i.e. $\hat{\mu}(f(\sigma))=\mu(f(T \sigma))$ ]. The following lemma will be used to prove the $T$-invariance of the Gibbs states supported by $\Omega_{1}$.

Lemma 3. Let $\mu \in \operatorname{Ext} \Delta$. If $\mu\left(\Omega_{1}\right)=1$ then for $\mu \times \hat{\mu}$-a.e. $(\sigma, \hat{\sigma}) \in \Omega_{1} \times \Omega_{1}$ the two contours $\gamma=\gamma(\sigma)$ and $\hat{\gamma}=\gamma(\hat{\sigma})$ intersect infinitely often in both $\pi_{+}$and $\pi_{-}$.

That, $\mu \times \hat{\mu}$-almost surely, the contours $\gamma$ and $\hat{\gamma}$ come to within distance 1 infinitely often is a consequence of Lemma 2 . It might therefore be no surprise that the remaining gap would also be closed an infinite number of times.

Proof of Lemma 3. Since the above property is measurable at infinity, if it fails then with $\mu \times \hat{\mu}$-probability $1 \gamma$ and $\hat{\gamma}$ intersect at only a finite number of times. This would imply for

$$
G_{n}=\left\{(\sigma, \hat{\sigma}) \mid \gamma(\sigma) \cap \gamma(\hat{\sigma}) \cap\left\{i_{x}>n\right\} \neq \emptyset\right\}
$$

that given any $\delta>0$ there is some $N=N(\delta)$ such that for any $n \geqq N$

$$
\mu \times \hat{\mu}\left(G_{n}\right)<\delta .
$$

Let however, $i_{n}$ be the earliest point in $\left\{i \in \mathbb{Z}^{2} \mid i_{x}>n\right\}$, in the lexicographic order, with the property that both $\gamma$ and $\hat{\gamma}$ intersect the $10 \times 10$ square centered at $i$; and let $B_{n}$ be the corresponding square. By Lemma $2 i_{n}$ is well defined for 
$\mu \times \hat{\mu}$ a.e. $(\sigma, \hat{\sigma})$. For a fixed $n$ we regard as essential parts of $\gamma$ and $\hat{\gamma}$ only their infinite connected components in $\mathbb{Z}^{2} / B_{n}$. It is easy to see that one may define a transformation $R$ which changes the spins of $(\sigma, \hat{\sigma})$ only in the corresponding set $B_{n}$, yielding configurations for which:

(i) $\gamma$ intersects $\hat{\gamma}$ in $B_{n}$

(ii) $\gamma$ and $\hat{\gamma}$ have no nonessential components in $\mathbb{Z}^{2} / B_{n}$.

The second property ensures that for $\mu \times \mu$-a.e. $(\sigma, \hat{\sigma})$ :

$$
\left|i_{n}(R((\sigma, \hat{\sigma})))-i_{n}((\sigma, \hat{\sigma}))\right| \leqq 2 \cdot 10
$$

and hence

$$
\text { card. } R^{-1}(\{(\sigma, \hat{\sigma})\}) \leqq(2 \cdot 10)^{2} 2^{10 \cdot 10} \equiv g .
$$

The uniform bound (3.5) and the fact that $R$ changes $(\sigma, \hat{\sigma})$ only in $B_{n}$ imply (by the DLR condition) that $\forall A \subset \Omega_{1} \times \Omega_{1}$

$$
\mu \times \hat{\mu}\left(R^{-1}(A)\right)<\varepsilon^{-1} \mu(A)
$$

with

$$
\varepsilon^{-1}=g \exp (4 \cdot 10 \cdot 10 \beta) .
$$

By (i) the range of $R$ is contained in $G_{n}$, thus

$$
\mu \times \hat{\mu}\left(R^{-1}\left(G_{n}\right)\right)=1
$$

and, by (3.7),

$$
\mu \times \hat{\mu}\left(G_{n}\right)>\varepsilon .
$$

(3.9) contradicts (3.4) and, therefore, proves the claim.

Lemma 4. If $\mu \in \operatorname{Ext} \Delta$ and $\mu\left(\Omega_{1}\right)=1$, then $\mu$ is invariant under the translation $T$.

Proof. Let $\mu$ satisfy the assumptions and let $\hat{\mu}=T \mu$. Without the loss of generality we may assume that the spins immediately below $\gamma$ are $\mu$-almost surely + .

Notice that if for some $(\sigma, \hat{\sigma}) \in \Omega_{1} \times \Omega_{1}$ there is an infinite cluster on which $\sigma=-1$ and $\hat{\sigma}=+1$ then, by the uniqueness of the infinite contour in $\Omega_{1}$, this cluster has to lie "above" $\gamma(\sigma)$ and "below" $\gamma(\hat{\sigma})$. This, however, is not possible if $\gamma(\sigma)$ and $\gamma(\hat{\sigma})$ intersect infinitely often in both directions.

Lemma 3 implies, therefore, that for $\mu \times \hat{\mu}$-a.e. $(\sigma, \hat{\sigma})$ there is no infinite cluster on which $\sigma>\hat{\sigma}$.

Let now $\Lambda$ be a finite box. Then, by the above conclusion, $\mu \times \hat{\mu}$-almost surely there exists a *cluster which completely surrounds $\Lambda$, on which $\sigma \leqq \hat{\sigma}$. For any finite $\bar{\Lambda} \supset \Lambda$ let $\alpha_{\bar{A}, \Lambda}(\sigma, \hat{\sigma})$ be the outermost such *cluster in $\bar{\Lambda}$, if such a set exists, and $\alpha_{\bar{\Lambda}, \Lambda}(\sigma, \hat{\sigma})=\emptyset$ otherwise. We shall also denote by $\tilde{\alpha}_{\Lambda, \Lambda}$ the complement, in $\bar{\Lambda}$, of the region surrounded by $\alpha_{\bar{\Lambda} . A}$. The key observation here is that ${ }^{1} \forall \Lambda \subset \bar{\Lambda}$ : Thus

$$
\left\{(\sigma, \hat{\sigma}) \in \Omega \times \Omega \mid \tilde{\alpha}_{\bar{\Lambda}, \Lambda}(\sigma, \hat{\sigma})=V\right\} \in \mathfrak{B}_{V \times V} .
$$

1 (4.7) can be viewed as defining a multidimensional generalization to set valued functions of the notion of nonanticipatory stopping time, which is very useful in the theory of martingales. 
Therefore, the conditional expectations of any measure $v$ on $\Omega \times \Omega$ satisfy:

$$
\begin{aligned}
& v\left(\cdot \mid \alpha_{\bar{\Lambda}, \Lambda} \neq \emptyset\right) v\left(\left\{\alpha_{\bar{\Lambda}, \Lambda}(\cdot) \neq \emptyset\right\}\right) \\
& \quad=\sum_{V \subset \bar{\Lambda}} \sum_{\substack{(\sigma, \hat{\sigma}) \text { s.t. } \\
\tilde{\alpha}_{\bar{\Lambda}, \Lambda}(\sigma, \hat{\sigma})=V}} v\left(\cdot \mid(\sigma, \hat{\sigma})_{V \times V}\right) v_{V \times V}\left(\left\{(\sigma, \hat{\sigma})_{V \times V}\right\}\right) .
\end{aligned}
$$

Consider now $f \in \mathfrak{B}$ which is bounded and monotone in the FKG sense. For the case $v=\mu \times \hat{\mu}$, the factorization and the Markov property of the DLR conditions imply for each of the terms in the above sum:

$$
\mu \times \hat{\mu}_{\Lambda \times \Lambda}\left(\cdot \mid(\sigma, \hat{\sigma})_{V \times V}\right)=\mu_{\Lambda}\left(\cdot \mid \sigma_{\alpha_{\bar{\Lambda}, \Lambda}}\right) \times \hat{\mu}_{\Lambda}\left(\cdot \mid \hat{\sigma}_{\alpha_{\bar{\Lambda}, \Lambda}}\right) .
$$

Since only terms with

$$
\sigma_{\alpha_{\bar{\Lambda}, \Lambda}} \leqq \hat{\sigma}_{\alpha_{\bar{\Lambda}, \Lambda}}
$$

contribute, Proposition 2 (the FKG inequality) implies that for any monotone $f$

$$
\mu \times \mu\left(f(\sigma) \mid \alpha_{\bar{\Lambda}, \Lambda} \neq \emptyset\right) \leqq \mu \times \mu\left(f(\hat{\sigma}) \mid \alpha_{\bar{\Lambda}, \Lambda} \neq \emptyset\right) .
$$

Thus

$$
\begin{aligned}
\mu(f(\cdot)) & \equiv \mu \times \hat{\mu}(f(\sigma)) \\
& =\lim _{\bar{\Lambda} \mathbb{Z}^{2}} \mu \times \hat{\mu}\left(f(\sigma) \mid \alpha_{\bar{\Lambda}, \Lambda} \neq \emptyset\right) \quad \mu \times \hat{\mu}\left(\left\{\alpha_{\bar{\Lambda}, \Lambda} \neq \emptyset\right\}\right) \\
& \leqq \lim _{\bar{\Lambda}>\mathbb{Z}^{2}} \mu \times \hat{\mu}\left(f(\hat{\sigma}) \mid \alpha_{\bar{\Lambda}, \Lambda} \neq \emptyset\right) \quad \mu \times \hat{\mu}\left(\left\{\alpha_{\bar{\Lambda}, \Lambda} \neq \emptyset\right\}\right) \\
& =\mu \times \hat{\mu}(f(\hat{\sigma})) \equiv \hat{\mu}(f(\cdot)) .
\end{aligned}
$$

By the generality of $f$ :

$$
\hat{\mu} \underset{\mathrm{FK} G}{\gtrless} \mu
$$

and by a similar argument

$$
\mu \underset{\mathrm{FKG}}{\geqq} \hat{\mu} \text {. }
$$

Thus $\mu=\hat{\mu}$, which proves the translation invariance of $\mu$.

Proof of Proposition 5. Let $\mu$ be an extremal Gibbs state and let us assume that (4.1) is not satisfied. Since $\Omega_{1} \in \mathfrak{B}_{\infty}$, it follows by that

$$
\mu\left(\Omega_{1}\right)=1 \text {. }
$$

In particular, $\ell_{0}(\sigma)$ - the level of the lowest intersection of $\gamma(\sigma)$ with $\left\{i_{x}=0\right\}$, is well defined for $\mu$-a.e. $\sigma$ and has some probability distribution on $\mathbb{R}$, given by $\mu\left(\left\{\sigma \mid \ell_{0}(\sigma)\right.\right.$ $\leqq y\}$ ). This, however, is not possible since by Lemma 3 the above distribution should be translation invariant.

(4.1) holds therefore for any extremal Gibbs state and, by means of the decomposition to extremal elements (Proposition 1), for all the Gibbs states.

\section{Reduction of the General Case}

Let

$\Omega_{2}=\{\sigma \in \Omega \mid$ in $\sigma$ there is at least one infinite contour $\}$. 
In this section, the general case would be reduced to those studied already in the two previous sections by proving:

Proposition 6. $\forall \mu \in \Omega$,

$$
\mu\left(\Omega_{2} \backslash \Omega_{1}\right)=0 \text {. }
$$

While the results obtained so far relied essentially on the FKG inequality, in addition of course to the basic features of the system which make the notion of contours so useful, our analysis would now require the following additional property of the f.I.s.

Proposition 7 ([11]). Let $V_{n} \nearrow \mathbb{Z}^{n}$ be a sequence of finite regions which are symmetric with respect to the reflection $(x, y) \mapsto(x, 1-y)$. Then the Gibbs states $\mu_{n}^{ \pm}$which are induced in $V_{n}$, at some fixed temperature, by the boundary conditions

$$
\left.\sigma_{(x, y)}\right)=\left\{\begin{array}{ll}
-1 & y \geqq 0 \\
+1 & y<0
\end{array} \forall(x, y) \notin V_{n}\right.
$$

converge, and

$$
\lim _{n \rightarrow \infty} \mu^{ \pm}=\left(\mu_{+}+\mu_{-}\right) / 2 \text {. }
$$

We shall now denote $\Lambda_{n}=[-n, n] \times[-n, n]$. A useful corollary of Propositions 7 and 3 is :

\section{Lemma 5. Let}

$$
\theta_{m, n}=\left\{\sigma \in \Omega \mid \sigma \text { has } a-\text { cluster which connects } \Lambda_{n} \text { with } \Lambda_{m}^{c}\right\} .
$$

Then $\forall n, \varepsilon>0 \quad \exists m(n, \varepsilon)<\infty$ such that

$$
\mu_{V}^{ \pm}\left(\theta_{m, n}\right) \leqq \frac{1}{2}+\varepsilon
$$

for every symmetric $V \supset \Lambda_{m}$, with $\mu_{V}^{ \pm}$being defined as in Proposition 7.

Using Proposition 7, L. Russo proved what amounts to:

Lemma 6 ([13] - Lemma 13 combined with Proposition 4). Let $\mu \in \operatorname{Ext} \Delta \backslash\left\{\mu_{+, \beta}, \mu_{-, \beta}\right\}_{\beta}$. Then $\mu$-a.e. $\sigma$ has exactly one $\infty$ contour in $\pi_{-}$.

Proof of Proposition 6. It clearly suffices to prove (5.1) for $\mu \in \operatorname{Ext} \Delta(\beta) \backslash\left\{\mu_{+, \beta}, \mu_{-, \beta}\right\}$. Let $\mu$ be such a state. We shall prove (5.1) by showing that

$$
\mu\left(\Omega_{2} \backslash \Omega_{1}\right) \leqq 3 / 4
$$

which, by Proposition 1 , is sufficient since $\Omega_{2} \backslash \Omega_{1} \in \mathfrak{B}_{\infty}$.

By Lemma 6, $\mu$-a.e. $\sigma$ has a unique contour which has an infinite connected piece in $\pi_{-}$. We denote it by $\delta(\sigma)$, and the two regions on its + and - sides by $D_{+}(\sigma), D_{-}(\sigma) \subset \mathbb{Z}^{2}$.

Claim. The conditional $\mu$-probability of there being another $\infty$ contour in $D_{+}$ conditioned on:

(i) the position of $\delta(\sigma)$

(ii) $\mathfrak{B}_{D_{-}(\sigma)}$

is, uniformly, $\leqq \frac{1}{2}$. 
Proof of the Claim. WLOG we assume that the spins "below" $\delta(\sigma)$ are $+\mu$-almost surely.

Let $n, \varepsilon>0$ and $m=m(n, \varepsilon)$. By the uniqueness expressed in Lemma 6, for $\mu$-a.e. $\sigma$ there are + clusters in $D_{+}(\sigma) \cap \pi_{-} \Lambda_{m}$ and - clusters in $D_{-}(\sigma) \cap \pi_{-} \Lambda_{m}$ which connect $\delta(\sigma)$ with the $\left\{i_{x}=0\right\}$ line. There is therefore $h \geqq m$ large enough so that with $\mu$-probability $\geqq(1-\varepsilon)$ such + and - clusters exist in $\pi_{-} \cap \Lambda_{h}$. Let us denote the outermost such contours in $\pi_{-} \cap \Lambda_{h}$ by $\tau_{+}$and $\tau_{-}$, including in $\tau_{+}$the shortest piece along $\delta(\sigma)$ which connects it with $\tau$, and let $\bar{\tau}$ be the complement in $\pi_{-}$of the set enclosed by $\tau_{-} \tau_{+}$and the $\left\{i_{x}=0\right\}$ line.

Consider now the $\mu$-probability that in $D_{+}$there is a - cluster connecting $\Lambda_{n}$ to $\Lambda_{m}$, conditioned:

(i) the position of $\delta(\sigma)$

(ii) $\mathfrak{B}_{D_{-}(\sigma)}$

(iii) $\mathfrak{B}_{\Lambda_{h}^{c}}$

(iv) the position of $\tau_{+}$.

By the FKG inequality and the Markov property, this probability only increases when the +1 boundary provided by the spins along $\delta(\sigma)$ is replaced by +1 boundary condition along $\tau_{-}$, which is further withdrawn into $D_{-}(\sigma)$, and -1 along the reflection of $\tau_{+} \cup \tau_{-}$with respect to the $\left\{i_{x}=\frac{1}{2}\right\}$ line. The above probability is therefore uniformly smaller than $\mu_{V}^{ \pm}\left(\theta_{n, m}\right) ; V$ being the volume enclosed by $\tau_{+} \cup \tau_{-}$and its reflection. [Using the "nonanticipatory" property of $V^{c}$ defined, for $\tilde{\alpha}_{\bar{\Lambda}, \Lambda}$, by (4.7).]

Using Lemma 5 , averaging over $\mathfrak{B}_{D_{+} \backslash \Lambda s}$ and $\tau_{+}$, and letting $\varepsilon \rightarrow 0$ we conclude that the probability of there being an $\infty$ - cluster in $D_{+}$conditioned on (5.6) is at most $\frac{1}{2}$. This proves the claim.

A direct consequence is that the $\mu$-probability of $\delta(\sigma)$ being the only $\infty$ contour of $\sigma$ is at least $\frac{1}{2} \frac{1}{2}=\frac{1}{4}$. However, the number of $\infty$ contours is measurable at infinity

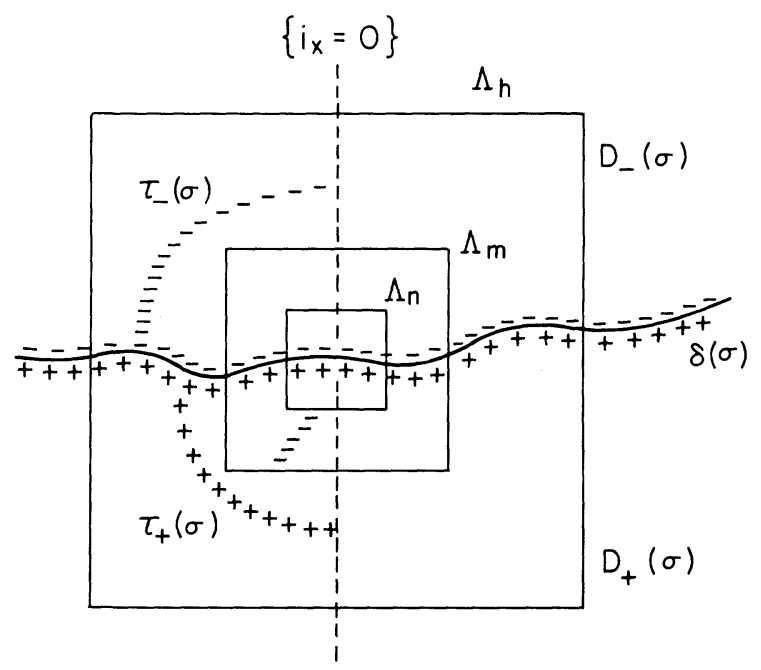

Fig. 1. A reference for the notation used in the proof of Proposition 6 
and thus, by the extremality of $\mu$,

$$
\mu(\{\sigma \in \Omega \mid \sigma \text { has exactly one } \infty \text { contour }\})=1 .
$$

Let now $k \in \mathbb{Z}$. By Lemma 6 , $\mu$-a.e. $\sigma$ has exactly one $\infty$ contour in each of the regions $\left\{i_{x} \geqq 2|k|\right\},\left\{i_{x} \leqq-2|k|\right\} \subset \mathbb{Z}^{2}$. If such $\sigma$ has only one $\infty$ contour then the above two are parts of it and, in particular, they are connected by a finite contour piece. This proves that the $\infty$ contour's intersection with $\left\{i_{x}=k\right\}$ is both finite and nonempty. By the countable additivity of $\mu$, for $\mu$-a.e. $\sigma$ the above is true for any $k \in \mathbb{Z}$, which implies (5.1).

Placing the three marbles together we have a proof of the main result of this paper.

Proof of Theorem 1. Let $\mu \in \Delta(\beta)$. Then, using Propositions 5 and 6 :

$$
\mu\left(\Omega_{2}\right) \leqq \mu\left(\Omega_{2} \backslash \Omega_{1}\right)+\mu\left(\Omega_{1}\right)=0 .
$$

Therefore the criterion provided by Proposition 4 is applicable. It implies:

$$
\mu \in \operatorname{span}\left\{\mu_{+, \beta}, \mu_{-, \beta}\right\} \text {. }
$$

\section{Remarks on Extensions}

1. The instability of phase coexistence may be a generic feature of two dimensional short range systems. The partial results of Sects. 3 and 4 can be extended to other two-phase systems for which contour arguments and the FKG inequality are applicable, e.g. the Widom-Rowlinson lattice model which was studied in $[6,7]$. In fact, the method of Sect. 4 yields a stronger result; that if, for some $\mu \in \Delta, \mu$-a.e. $\sigma$ has a finite number of oocontours and if these may somehow be "labeled at infinity" (which would be the case if they wind only finite numbers of times around the origin), then $\mu$ is translation invariant. In Sect. 5 additional properties of the f.I.s. were invoked (Proposition 7). For some models which do not have these properties, as in the above mentioned case, the instability of phase coexistence may still be proven for low temperatures using that stronger result in conjunction with Peierls-type arguments. The latter have the advantage of generalizability, at the expense of the restriction to low temperatures.

2. It is suspected that the phase coexistence which occurs in three dimensions destabilizes at some temperature below $T_{c}$, dubbed the "roughening temperature". It would be very interesting to see a further development of the stochastic geometrical methods which might shed some light on this phenomenon.

Acknowledgements. It is a pleasure to thank Joel L. Lebowitz and Barry Simon for stimulating discussions.

\section{References}

1. Dobrushin, R.L.: Theor. Prob. Appl. 17, 582 (1972)

2. Dobrushin, R.L.: Theor. Prob. Appl. 13, 197 (1968)

Lanford, O.E. III, Ruelle, D.: Commun. Math. Phys. 13, 194 (1969)

3. Fortuin, C.M., Kasteleyn, P.W., Ginibre, J.: Commun. Math. Phys. 22, 89 (1971) 
4. Holley, R.: Commun. Math. Phys. 36, 227 (1974)

5. van Beijeren, H.: Commun. Math. Phys. 40, 1 (1975)

6. Lebowitz, J.L., Monroe, J.L.: Commun. Math. Phys. 28, 301 (1972)

7. Bricmont, J., Lebowitz, J.L., Pfister, C.E., Olivieri, E.: Commun. Math. Phys. 66, 1 (1979)

8. Gallavotti, G.: Commun. Math. Phys. 27, 103 (1972)

9. Abraham, D.B., Reed, P.: Phys. Rev. Lett. 33, 377 (1974)

10. Abraham, D.B., Reed, P.: Commun. Math. Phys. 49, 35 (1976)

11. Messager, A., Miracle-Sole, S.: J. Stat. Phys. 17, 245 (1977)

12. Coniglio, A., Nappi, C., Peruggi, F., Russo, L.: Commun. Math. Phys. 51, 315 (1976)

13. Russo, L.: Commun. Math. Phys. 67, 251 (1979)

14. Aizenman, M.: Phys. Rev. Lett. 43, 407 (1979)

15. Merlini, D.: Preprint

16. Higuchi, Y.: In: Proc. Colloquium on Random Fields (Esztergom, June 1979). To appear

Communicated by A. Jaffe

Received June 18, 1979; in revised form December 13, 1979 